\title{
EFEITOS DO AUTOCUIDADO APOIADO POR ENFERMEIROS EM HOMENS COM DIABETES MELLITUS TIPO 2
}

\author{
EFFECTS OF SELF-CARE SUPPORTED BY NURSES IN \\ MEN WITH TYPE 2 DIABETES MELLITUS
}

\section{EFECTOS DEL AUTOCUIDADO APOYADO POR ENFERMERAS EN HOMBRES CON DIABETES MELLITUS TIPO 2}

\author{
Guilherme Oliveira de Arruda ${ }^{1}$ \\ Sonia Silva Marcon ${ }^{2}$ \\ Hellen Emília Peruzzo Aveiro ${ }^{3}$ \\ Maria do Carmo Fernandez Lourenço Haddad ${ }^{4}$ \\ Luciana Puchalski Kalinke \\ Gleiciane da Silva Fonseca ${ }^{6}$ \\ Afonso de Arruda Martinhago ${ }^{7}$
}

Como citar este artigo: Arruda GO, Marcon SS, Aveiro HEP, Haddad MCFL, Kalinke LP, Fonseca GS, et al. Efeitos do autocuidado apoiado por enfermeiros em homens com Diabetes Mellitus tipo 2. Rev baiana enferm. 2022:36:e43380.

Objetivo: avaliar os efeitos do autocuidado apoiado desenvolvido por enfermeiros sobre o conhecimento referente à doença, autocuidado, ajustamento psicológico e autoeficácia de homens com Diabetes Mellitus tipo 2. Método: ensaio clínico randomizado (REBEC: RBR-46zk89; ClinicalTrials: NCT02974413), que envolveu dez clusters, 73 homens de 40 a 70 anos, com Diabetes Mellitus tipo 2, distribuídos nos Grupos Intervenção (n=30) e Controle $(n=43)$. Intervenção realizada individualmente ou em grupo. Utilizaram-se Equações de Estimações Generalizadas para comparações intra e intergrupos. Resultados: efeito estatisticamente significante só foi observado em relação ao aumento do conhecimento sobre a doença $(\mathrm{p}<0,001)$, especialmente na participação individual ( $\mathrm{p}=0,005)$. Referente ao tipo de participação, evidenciou-se, de forma não significante, melhor ajustamento psicológico e autocuidado para os participantes da intervenção em grupo. Conclusão: o autocuidado apoiado por enfermeiro melhorou o conhecimento de homens com Diabetes Mellitus tipo 2 e, de forma não significante, promoveu ajustamento psicológico e autocuidado na participação em grupo.

Descritores: Saúde do homem. Diabetes Mellitus. Autocuidado. Atenção Primária à Saúde. Enfermagem.

Objective: to evaluate the effects of supported self-care developed by nurses on knowledge related to disease, self-care, psychological adjustment and self-efficacy of men with type 2 Diabetes Mellitus. Method: randomized clinical trial (REBEC: RBR-46zk89; ClinicalTrials: NCTO2974413), which involved ten clusters, 73 men aged 40 to 70 years, with Type 2 Diabetes Mellitus, distributed in the Intervention $(n=30)$ and Control $(n=43)$ groups. Intervention performed

\footnotetext{
Enfermeiro. Doutor em Enfermagem. Professor da Universidade Federal do Mato Grosso do Sul. Coxim, Mato Grosso do Sul, Brasil. enfgoa@gmail.com. https://orcid.org/0000-0003-1690-4808.

Enfermeira. Doutora em Filosofia da Enfermagem. Professora Titular da Universidade Estadual de Maringá. Maringá, Paraná, Brasil. https://orcid.org/0000-00026607-362X.

Doutora em Enfermagem. Professora da Universidade Estadual do Paraná. Paranavaí, Paraná, Brasil. https://orcid.org/0000-0002-0786-0447.

Enfermeira. Doutora em Enfermagem. Professora Sênior da Universidade Estadual de Londrina. Londrina, Paraná, Brasil. https://orcid.org/0000-000 I-7564-8563.

Enfermeira. Doutora em Ciências da Saúde. Professora da Universidade Federal do Paraná. Curitiba, Paraná, Brasil. https://orcid.org/0000-00034868-8193.

Enfermeira. Especialista em Atenção à Saúde da Criança e Adolescente. Universidade Federal do Mato Grosso do Sul. Campo Grande, Mato Grosso do Sul, Brasil. https://orcid.org/0000-000 | -7450- I 375.

Estudante de Medicina. Universidad Sudamericana. Salto del Guairá, Paraguai. https://orcid.org/0000-0003-2628-95 I I.
} 
individually or in groups. Generalized Estimator equations were used for intra- and intergroup comparisons. Results: statistically significant effect was only observed in relation to increased knowledge about the disease $(p<0.001)$, especially in individual participation ( $p=0.005)$. Regarding the type of participation, in a non-significant way, better psychological adjustment and self-care for the participants of the group intervention. Conclusion: the self-care supported by nurses improved the knowledge of men with type 2 Diabetes Mellitus and, in a non-significant way, promoted psychological adjustment and self-care in-group participation.

Descriptors: Men's health. Diabetes Mellitus. Self-care. Primary Health Care. Nursing.

Objetivo: evaluar los efectos del autocuidado apoyado desarrollado por las enfermeras sobre el conocimiento relacionado con la enfermedad, el autocuidado, el ajuste psicológico y la autoeficacia de los hombres con Diabetes Mellitus tipo 2. Método: ensayo clinico aleatorizado (REBEC: RBR-46zk89; ClinicalTrials: NCT02974413), que involucró a diez clusters, 73 hombres de 40 a 70 años, con Diabetes Mellitus Tipo 2, distribuidos en los grupos de Intervención $(n=30)$ y Control $(n=43)$. Intervención realizada individualmente o en grupos. Se utilizaron ecuaciones de estimación generalizada para las comparaciones intra e intergrupales. Resultados: sólo se observó efecto estadísticamente significativo en relación con un mayor conocimiento sobre la enfermedad $(p<0,001)$, especialmente en la participación individual $(p=0,005)$. En cuanto al tipo de participación, se evidenció, de manera no significativa, un mejor ajuste psicológico y autocuidado para los participantes de la intervención grupal. Conclusión: el autocuidado apoyado por enfermeras mejoró el conocimiento de los hombres con Diabetes Mellitus tipo 2 y, de manera no significativa, promovió el ajuste psicológico y el autocuidado en la participación grupal.

Descriptores: Salud de los Hombres. Diabetes Mellitus. Autocuidado. Atención Primaria de Salud. Enfermería.

\section{Introdução}

O número de casos de Diabetes Mellitus (DM) aumenta consideravelmente no mundo. Estima-se que, até 2035, mais de 590 milhões de pessoas conviverão com esta condição crônica ${ }^{(1)}$. A fim de facilitar o envolvimento espontâneo das pessoas com a tomada de decisões, sugere-se o desenvolvimento de uma educação que privilegie o empoderamento para o autocuidado em $\mathrm{DM}^{(2)}$. Destaca-se o Autocuidado Apoiado (AA), que pode ser definido como a aplicação de ações articuladas que promovam a autogestão em saúde. O AA é perfeitamente aplicável às pessoas que convivem com DM e pode ser organizado com base no método de gestão dos "5 As": Avaliação, Aconselhamento, Acordo, Assistência e Acompanhamento ${ }^{(3)}$.

Para a organização e operacionalização dos processos mentais de mudança comportamental, e o estabelecimento de metas para controle do diabetes, propõe-se que o Protocolo de Mudança de Comportamento seja implementado alinhado às fases do AA. O protocolo é constituído de cinco etapas: exploração do problema, esclarecimento de sentimentos e significados, definição de metas, comprometimento com a ação e avaliação da experiência e do plano de autocuidado ${ }^{(4)}$.
Embora já existam estudos realizados no Brasil, propondo intervenções educativas para a atenção às pessoas com diabetes, ainda são escassos os que avaliam essas intervenções na Atenção Primária à Saúde (APS) ${ }^{(5)}$. Esta escassez é ainda maior em relação aos estudos realizados especificamente com homens. A população masculina é vulnerável do ponto de vista epidemiológico e sociocultural, visto que os homens, em geral, apresentam altos índices de comportamentos prejudiciais à saúde ${ }^{(6)}$. Inclusive, já foi identificado que os homens com diagnóstico de diabetes aderem menos a comportamentos saudáveis, têm menor controle alimentar e não realizam exames laboratoriais de rotina ${ }^{(7)}$.

Estudo de intervenção mostrou que, além de homens e mulheres utilizarem estratégias de enfrentamento diferentes, eles aderem com maior frequência à prática de atividade física, mas carecem de orientações sobre a dieta, enquanto as mulheres estão mais inclinadas a adequarem a dieta ${ }^{(8)}$. Neste sentido, é importante destacar que as representações de masculinidade dominante marcam o pensar e o fazer do homem e reforçam atributos como força, destemor e invulnerabilidade. Isto, por sua vez, afasta-os de 
possibilidades de autocuidado e aproxima-os de comportamentos de risco, o que pode prejudicar seu estado de saúde ${ }^{(9)}$. Inclusive, os homens tendem a não valorizar comportamentos em saúde e nem mesmo a presença de fatores de risco para doenças cardiovasculares, conforme constatado em estudo realizado com metalúrgicos ${ }^{(10)}$.

Contudo, os enfermeiros que atuam no âmbito da APS podem interferir nessa realidade ajudando os homens a se aproximarem dos serviços de saúde e também a desenvolverem maior autossuficiência na gestão de suas condições de saúde ${ }^{(11)}$. Nesta direção, em estudo realizado nos Estados Unidos, os próprios homens indicaram a necessidade de programas educativos sobre o estilo de vida voltados ao cuidado em diabetes ${ }^{(12)}$. Ademais, já foram observados efeitos positivos, embora discretos, do autocuidado apoiado por enfermeiros sobre o comportamento em saúde e medidas antropométricas de homens com diabetes ${ }^{(13)}$. Contudo, observa-se uma lacuna na literatura e na prática assistencial, no que se refere às estratégias direcionadas aos homens que apresentam condições crônicas e efeitos sobre outros desfechos.

Frente às diferenças de gênero no cuidado em saúde e às representações de masculinidades que podem vulnerabilizar a saúde do homem com DM2, surge a necessidade de se investigar estratégias que contribuam para o autocuidado desse grupo populacional. Com isso, questiona-se: Quais os efeitos de uma intervenção pautada no AA por enfermeiros, junto a homens adultos com DM2 no âmbito da APS? Definiu-se como objetivo avaliar os efeitos do AA desenvolvido por enfermeiros sobre o conhecimento referente à doença, autocuidado, ajustamento psicológico e autoeficácia de homens com DM2.

\section{Método}

Ensaio clínico randomizado por clusters, controlado, paralelo e sem cegamento, com aplicação do referencial CONSORT. Realizado entre abril de 2016 e julho de 2018 em município do Noroeste do estado do Paraná, Sul do Brasil.
Foram adotados como critérios de inclusão: ser do sexo masculino, ter diagnóstico médico de DM2, idade entre 40 e 70 anos e estar cadastrado nas equipes de Estratégia Saúde da Família (ESF) em estudo. Os critérios de exclusão foram: apresentar lesões em órgãos-alvo ou comorbidades/complicações decorrentes do diabetes (tratamento hemodialítico, amaurose, sequelas incapacitantes de acidente vascular cerebral/insuficiência cardíaca e distúrbios psíquicos que comprometessem a participação no estudo). Consideraram-se os seguintes critérios de descontinuidade: óbito, mudança para área sem cobertura da ESF ou outro município, problema grave de saúde que impossibilitasse tomar parte no estudo e desejo de interromper a participação.

Para o cálculo do tamanho da amostra, elegeu-se nível de significância de $5 \%(\alpha=0,05)$ e poder do teste de $80 \%(\beta=0,20)$, com nível de confiança de 95\%, valor da diferença mínima detectável (1,0\% do valor da hemoglobina glicada) e desvio padrão da média de hemoglobina glicada de 2,0\%. Estimou-se, assim, o tamanho amostral mínimo de 128 homens.

O recrutamento dos participantes foi realizado por meio de contatos prévios com as equipes de ESF, com a posterior distribuição de cartas-convite, elaboradas pelos pesquisadores, identificadas de forma personalizada com o nome dos homens, e entregues aos Agentes Comunitários de Saúde. Foram agendados e realizados encontros nas Unidades Básicas de Saúde (UBS), entre abril de 2016 e janeiro de 2017, para a sensibilização e o convite para os homens.

Passou-se à fase de randomização estratificada por clusters, que foi realizada sob suporte de profissional de estatística. Definiu-se, por cluster, cada uma das 10 UBS onde os homens foram convidados. As UBS a serem alocadas no GC e no GI foram determinadas por meio da técnica de estatística multivariada denominada Cluster Analysis, tomando-se como referência as médias de desvio padrão de hemoglobina glicada por UBS. Desse modo, as UBS foram aglomeradas de acordo com a similaridade dos valores de hemoglobina glicada, de maneira a atingir a homogeneização dos grupos, com alocação de cinco 
UBS em cada grupo. A aleatorização por agrupamentos (clusters) foi escolhida para evitar o viés de contaminação entre os grupos amostrais.

Durante os encontros de sensibilização, foram discutidos temas relacionados ao autocuidado, tais como: importância do autocuidado, alimentação saudável, atividade física, uso de medicamentos, monitorização glicêmica, resolução de problemas, redução dos riscos de complicações agudas e crônicas e aspectos psicossociais. Aos que aceitaram participar do estudo, foram aplicados os instrumentos de coleta de dados e aferidas pressão arterial e medidas antropométricas (peso, altura, circunferência da cintura e percentual de gordura corporal). Realizou-se, assim, a etapa de Avaliação do AA, com a coleta dos dados iniciais (aplicação de instrumentos sobre conhecimento, ajustamento psicológico, autoeficácia e autocuidado) e início do Aconselhamento. Os pesquisadores envolvidos na mensuração foram treinados previamente.

Os homens alocados no Grupo Intervenção (GI) foram contatados e agendou-se um segundo encontro, individual ou em grupo, para continuidade da intervenção orientada pelo $\mathrm{AA}^{(4)}$ e pelo Protocolo de Mudança de Comportamento. Entre o primeiro e o segundo encontro, foram realizados contatos telefônicos individualizados (ao menos dois), a fim de promover manutenção do vínculo, sanar dúvidas sobre a pesquisa e algum tema abordado no encontro inicial e, ainda, o agendamento do encontro seguinte. Iniciou-se, assim, a etapa do Acompanhamento, que fez parte do AA e foi implementado de forma transversal até o fim do seguimento.

O foco principal do segundo encontro recaiu sobre os hábitos alimentares saudáveis, porém dúvidas acerca de outros temas relacionados ao diabetes, identificadas na Avaliação, foram esclarecidas e contextualizadas com o cotidiano dos homens, a fim de que eles pudessem considerá-los como possíveis campos de atuação no autocuidado. Avançou-se, portanto, com a etapa de Aconselhamento do AA. Este segundo encontro foi realizado nas modalidades individual e em grupo, de acordo com as possibilidades dos homens.
Ainda nesse encontro, operacionalizaram-se as etapas de Acordo e de Assistência do AA, por meio da aplicação do Protocolo de Mudança de Comportamento, com foco na etapa de construção do Plano Inteligente de Cuidados e no estabelecimento de metas de autocuidado. Os homens foram contatados por telefone e convidados a participar dos grupos. Foram realizados cinco encontros (um por UBS), entre os meses de maio e junho de 2017, procedendo-se a um trabalho interativo, gerando discussões e exposição de experiências pessoais. Foram acionadas questões do Protocolo de Mudança de Comportamento para fomentar possibilidades de ações de autocuidado.

Para identificar tais ações, eram realizados questionamentos, como: Qual é a sua maior dificuldade para cuidar da sua saúde? Como você se sente com essa situação de ter de cuidar da sua saúde? O que você quer fazer para melhorar sua saúde? Como você pode mudar alguma coisa na sua vida para se sentir melhor? O que você acha que pode atrapalhar a conquista da sua meta? Tem alguma pessoa que possa lhe ajudar a alcançar sua meta?

Os homens que não puderam participar dos grupos nas UBS foram visitados no domicílio, onde se realizaram encontros individuais para a intervenção, entre os meses de outubro de 2017 e fevereiro de 2018. Destaca-se que 18 homens participaram dos encontros em grupo e outros 18 participaram dos encontros individuais. O terceiro encontro, individual, contou com ações que fizeram parte da etapa de Acompanhamento, reavaliando-se as dúvidas e o levantamento de dificuldades na implementação do plano de cuidados, seguido da coleta de dados finais e de devolutiva sobre resultados de medidas e exames.

Os indivíduos que compuseram o Grupo Controle (GC) participaram do encontro inicial e receberam orientações, ao final do seguimento, acerca do conhecimento sobre o diabetes e ainda os resultados de exames laboratoriais e medidas antropométricas.

Os dados iniciais foram coletados pelo pesquisador principal e mais dois pesquisadores 
assistentes, mediante aplicação de instrumentos de coleta de dados e verificação de medidas. Os dados finais foram coletados por duplas de pesquisadores que não participaram da intervenção e nem da coleta dos dados iniciais. Foram elencados como desfechos primários para o presente estudo: o conhecimento sobre o diabetes, o autocuidado, as atitudes frente à doença (ajustamento psicológico) e a autoeficácia em diabetes. Para mensuração dos referidos desfechos, foram aplicados os seguintes instrumentos: Questionário sobre Conhecimento em Diabetes (DKN-A), Questionário de Atividades de Autocuidado com o Diabetes (QAD), Questionário de Atitudes frente ao Diabetes (ATT-19) ${ }^{(14)}$ e Escala de Autoeficácia em Diabetes - Versão Curta (EAD-VC) ${ }^{(15)}$.

$\mathrm{Na}$ comparação das mesmas variáveis para grupos distintos, utilizou-se o teste de Qui-quadrado de Pearson. Para a comparação dos escores referentes ao conhecimento, autocuidado, atitudes e autoeficácia (variáveis dependentes), optou-se pelo uso de modelos de Equações de Estimações Generalizadas (EEG). Para tanto, adotou-se o tipo de resposta linear (função de ligação identidade), o estimador da matriz de covariância robusto e uma matriz de correlação trabalho não estruturada. Utilizou-se comparações por pares com o ajuste de Bonferroni. O Grupo (Intervenção e Controle) e o Momento de Observação (1ำ e $2^{\circ}$ momentos de observação) foram aplicados como variáveis independentes para a análise dos efeitos principais (efeito de uma variável independente sobre a dependente) e dos efeitos de interação (efeito combinado das duas variáveis independentes sobre a dependente). Adotou-se ainda o tipo de participação na intervenção (individual ou grupo) como covariável para se analisar as possíveis diferenças atribuídas a esta variável. Foram estimados média, erro padrão, diferença média e intervalo de confiança de $95 \%$. O nível de significância pré-fixado foi de 5\% e p-valor para a análise de EEG foi obtido por meio do qui-quadrado de Wald.

O projeto de pesquisa foi apreciado e aprovado pelo Comitê Permanente de Ética em Pesquisas com Seres Humanos da
Universidade Estadual de Maringá e o protocolo de intervenção registrado (REBEC: RBR-46zk89; ClinicalTrials: NCT02974413).

\section{Resultados}

Dos 39 participantes que eram do GI e foram descontinuados do estudo, 20 saíram antes da intervenção propriamente: 7 deles não foram localizados por meio do contato informado no momento do convite, 6 desistiram por desinteresse autodeclarado, 2 por óbito, 2 por terem sofrido Acidente Vascular Cerebral e 3 por outros motivos (início de tratamento para câncer, mudança de cidade e participação em outro estudo clínico). Dos 19 homens que foram descontinuados entre o primeiro e o segundo encontro da intervenção: 10 desistiram por diferentes motivos, 3 não foram encontrados pelos contatos informados, 2 mudaram-se do município, 2 sofreram eventos vasculares (infarto e início de tratamento hemodialítico), 1 por óbito (infarto) e 1 não foi encontrado no domicílio para o encontro de intervenção.

No GI, não foram verificadas diferenças quanto aos dados da linha de base para variáveis sociodemográficas, comportamentais e clínicas, entre os homens que concluíram o estudo, conforme o tipo de participação na intervenção (individual ou em grupo). Também não foram verificadas diferenças significantes na linha de base entre GI e GC. Contudo, destaca-se que a média de renda familiar ( $G I=2,709 \times \mathrm{GC}=2,903)$, a referência ao plano de saúde (GI=40,0\% x GC=44,2\%), à prática de atividade física (GI=46,7\% x GC=58,1\%) e os níveis de ajustamento psicológico (GI=49,6 x $G C=53,6)$ e autoeficácia ( $G I=3,7$ x $G C=3,8$ ) foram ligeiramente maiores no GC, que também apresentou menores níveis de hemoglobina glicada (GI $=8,9 \%$ x GC=8,1\%).

No GI, observou-se maior tempo médio de diagnóstico (GI=12,0 x GC=10,5) e frequências maiores de tabagismo ( $\mathrm{GI}=10,0 \%$ x GC=7,0\%), uso de bebidas alcóolicas (GI=46,7\% x GC=34,9\%), problemas renais ( $\mathrm{GI}=23,3 \% \times \mathrm{GC}=9,3 \%$ ), oftalmológicos (GI=80,0\% x GC=62,8\%) e de 
cicatrização (GI=16,7\% x $G C=16,3 \%)$. A frequência do conhecimento satisfatório no $2^{\circ}$ momento de observação foi maior no GI do que no GC $(93,3 \%>63,4 \%)$. Ademais, o grau satisfatório de conhecimento aumentou no GI após a aplicação da intervenção, passando de 55,1\% para $93,3 \%$.

O Fluxograma 1 apresenta o andamento dos Clusters e dos participantes do estudo.

Fluxograma 1 - Ensaio clínico randomizado, conforme CONSORT 2010

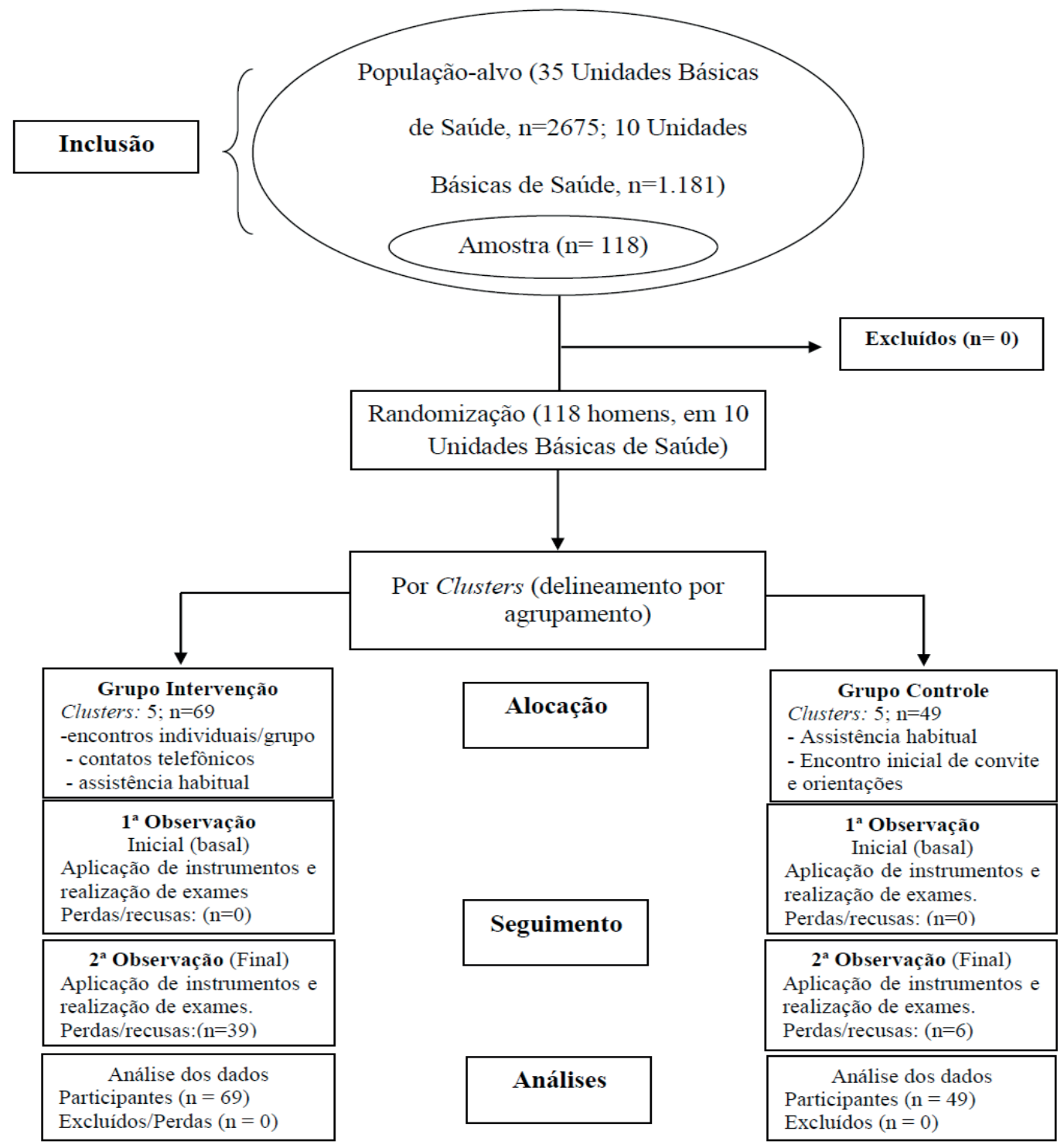

Fonte: Elaboração própria.

Os resultados do teste dos efeitos principais, com as variáveis grupo e momento de observação, e da interação entre essas variáveis, apontaram efeitos sobre conhecimento (grupo: $\mathrm{p}=0,048$; momento de observação: $\mathrm{p}=0,006$; e interação grupo/momento de observação: $\mathrm{p}=0,003$ ), ajustamento psicológico (momento de observação: p=0,001); e autoeficácia (grupo: $\mathrm{p}=0,022$ ); e momento de observação: $p=0,002$ ). As médias estimadas, erros-padrão e IC 95\% para GI e GC nos dois momentos de observação são apresentadas na Tabela 1 e representam a interação entre esses dois fatores para cada um dos desfechos estudados. 
Tabela 1 - Estimativas das médias de conhecimento, autocuidado, ajustamento psicológico e autoeficácia por grupos de homens com Diabetes Mellitus tipo II e momento de observação. Maringá, Paraná, Brasil - 2018. (N=73)

\begin{tabular}{|c|c|c|c|c|c|}
\hline \multirow{2}{*}{ Grupo } & \multirow{2}{*}{ Observação } & \multirow{2}{*}{ Média } & \multirow{2}{*}{ Erro padrão } & \multicolumn{2}{|c|}{ Intervalo de confiança de $95 \%$} \\
\hline & & & & Inferior & Superior \\
\hline \multicolumn{6}{|c|}{ Conhecimento } \\
\hline \multirow[t]{2}{*}{ Intervenção } & $1^{\circ}$ momento & 8,39 & 0,30 & 7,79 & 8,99 \\
\hline & $2^{\mathrm{o}}$ momento & 10,32 & 0,36 & 9,62 & 11,03 \\
\hline \multirow[t]{2}{*}{ Controle } & $1^{\mathrm{o}}$ momento & 8,59 & 0,39 & 7,82 & 9,36 \\
\hline & $2^{\mathrm{o}}$ momento & 8,52 & 0,42 & 7,69 & 9,35 \\
\hline \multicolumn{6}{|l|}{ Autocuidado } \\
\hline \multirow[t]{2}{*}{ Intervenção } & $1^{\circ}$ momento & 3,80 & 0,13 & 3,54 & 4,05 \\
\hline & $2^{\mathrm{o}}$ momento & 3,92 & 0,16 & 3,61 & 4,23 \\
\hline \multirow[t]{2}{*}{ Controle } & $1^{\mathrm{o}}$ momento & 3,69 & 0,15 & 3,39 & 3,99 \\
\hline & $2^{\mathrm{o}}$ momento & 3,73 & 0,16 & 3,41 & 4,04 \\
\hline \multicolumn{6}{|c|}{ Ajustamento Psicológico } \\
\hline \multirow[t]{2}{*}{ Intervenção } & $1^{\circ}$ momento & 52,93 & 1,29 & 50,40 & 55,45 \\
\hline & $2^{\mathrm{o}}$ momento & 49,43 & 1,63 & 46,23 & 52,63 \\
\hline \multirow[t]{2}{*}{ Controle } & $1^{\mathrm{o}}$ momento & 54,83 & 1,73 & 51,43 & 58,24 \\
\hline & $2^{\mathrm{o}}$ momento & 49,64 & 1,12 & 47,44 & 51,83 \\
\hline \multicolumn{6}{|l|}{ Autoeficácia } \\
\hline \multirow[t]{2}{*}{ Intervenção } & $1^{\mathrm{o}}$ momento & 3,64 & 0,06 & 3,52 & 3,76 \\
\hline & $2^{\mathrm{o}}$ momento & 3,78 & 0,07 & 3,64 & 3,91 \\
\hline \multirow[t]{2}{*}{ Controle } & $1^{\circ}$ momento & 3,75 & 0,08 & 3,59 & 3,90 \\
\hline & $2^{\mathrm{o}}$ momento & 3,98 & 0,04 & 3,90 & 4,06 \\
\hline
\end{tabular}

Fonte: Elaboração própria.

Na Tabela 2, fixou-se o momento de observação como marco para se comparar GI e GC em relação aos desfechos de interesse. Os resultados apontaram que não houve diferença significante entre os grupos no $1^{\circ}$ momento de observação para qualquer um dos desfechos. Por outro lado, no $2^{\circ}$ momento, verificou-se diferença significante em relação ao conhecimento (maior para o GI) e à autoeficácia (maior para o GC).

Tabela 2-Comparações de pares entre os grupos de homens com Diabetes Mellitus tipo II fixando-se o momento de observação para conhecimento, autocuidado, ajustamento psicológico e autoeficácia. Maringá, Paraná, Brasil - 2018. (N=73)

\begin{tabular}{|c|c|c|c|c|c|c|}
\hline \multirow{2}{*}{$\begin{array}{l}\text { Momento de } \\
\text { observação }\end{array}$} & \multirow{2}{*}{\multicolumn{2}{|c|}{ Grupos }} & \multirow{2}{*}{$\begin{array}{c}\text { Diferença } \\
\text { média(1) }\end{array}$} & \multicolumn{2}{|c|}{ Intervalo de confiança de $95 \%$} & \multirow{2}{*}{ p-valor $(2)$} \\
\hline & & & & Inferior & Superior & \\
\hline \multicolumn{7}{|l|}{ Conhecimento } \\
\hline $1^{\mathrm{o}}$ momento & Intervenção & Controle & $-0,20$ & $-1,17$ & 0,77 & 0,688 \\
\hline $2^{\circ}$ momento & Intervenção & Controle & 1,80 & 0,71 & 2,89 & 0,001 \\
\hline \multicolumn{7}{|l|}{ Autocuidado } \\
\hline $1^{\mathrm{o}}$ momento & Intervenção & Controle & 0,11 & $-0,29$ & 0,50 & 0,597 \\
\hline $2^{\mathrm{o}}$ momento & Intervenção & Controle & 0,19 & $-0,25$ & 0,64 & 0,391 \\
\hline \multicolumn{7}{|c|}{ Ajustamento Psicológico } \\
\hline $1^{\mathrm{o}}$ momento & Intervenção & Controle & $-1,90$ & $-6,15$ & 2,33 & 0,378 \\
\hline $2^{\mathrm{o}}$ momento & Intervenção & Controle & $-0,20$ & $-4,08$ & 3,67 & 0,918 \\
\hline \multicolumn{7}{|l|}{ Autoeficácia } \\
\hline $1^{\circ}$ momento & Intervenção & Controle & $-0,10$ & $-0,29$ & 0,09 & 0,303 \\
\hline $2^{\mathrm{o}}$ momento & Intervenção & Controle & $-0,20$ & $-0,36$ & $-0,04$ & 0,011 \\
\hline
\end{tabular}

Fonte: Elaboração própria.

(1) O sinal da diferença média corresponde à subtração: média do grupo intervenção - média do grupo controle.

(2) Teste post-hoc de Bonferroni. 
Quando fixado o tipo de Grupo, para se analisar as diferenças médias significantes entre os momentos de observação (Tabela 3), verificou-se que, para o GI, a pontuação média de conhecimento foi maior no $2^{\circ}$ momento; para o GC, a pontuação média de ajustamento psicológico foi menor e a de autoeficácia maior, no $2^{\circ}$ momento.

Tabela 3 - Comparações de pares entre os momentos de observação fixando-se o grupo de homens com Diabetes Mellitus tipo II para conhecimento, autocuidado, ajustamento psicológico e autoeficácia. Maringá, Paraná, Brasil - 2018. (N=73)

\begin{tabular}{|c|c|c|c|c|c|c|}
\hline \multirow[t]{2}{*}{ Grupo } & \multirow{2}{*}{\multicolumn{2}{|c|}{ Momentos de observação }} & \multirow{2}{*}{$\begin{array}{l}\text { Diferença } \\
\text { média(1) }\end{array}$} & \multicolumn{2}{|c|}{$\begin{array}{c}\text { Intervalo de } \\
\text { confiança de } 95 \%\end{array}$} & \multirow[t]{2}{*}{ p-valor (2) } \\
\hline & & & & Inferior & Superior & \\
\hline \multicolumn{7}{|c|}{ Conhecimento } \\
\hline Intervenção & $1^{\mathrm{o}}$ momento & $2^{\mathrm{o}}$ momento & 1,93 & $-2,70$ & $-1,17$ & $<0,001$ \\
\hline Controle & $1^{\mathrm{o}}$ momento & $2^{\mathrm{o}}$ momento & $-0,07$ & $-1,02$ & 1,15 & 0,905 \\
\hline \multicolumn{7}{|l|}{ Autocuidado } \\
\hline Intervenção & $1^{\mathrm{o}}$ momento & $2^{\mathrm{o}}$ momento & 0,12 & $-0,27$ & 0,52 & 0,542 \\
\hline Controle & $1^{\mathrm{o}}$ momento & $2^{\mathrm{o}}$ momento & 0,04 & $-0,33$ & 0,40 & 0,842 \\
\hline \multicolumn{7}{|c|}{ Ajustamento Psicológico } \\
\hline Intervenção & $1^{\circ}$ momento & $2^{\mathrm{o}}$ momento & $-3,49$ & $-7,32$ & 0,34 & 0,074 \\
\hline Controle & $1^{\mathrm{o}}$ momento & $2^{\mathrm{o}}$ momento & $-5,19$ & $-8,68$ & $-1,71$ & 0,003 \\
\hline \multicolumn{7}{|l|}{ Autoeficácia } \\
\hline Intervenção & $1^{\mathrm{o}}$ momento & $2^{\mathrm{o}}$ momento & 0,13 & $-0,03$ & 0,30 & 0,119 \\
\hline Controle & $1^{\mathrm{o}}$ momento & $2^{\mathrm{o}}$ momento & 0,23 & 0,07 & 0,40 & 0,005 \\
\hline
\end{tabular}

Fonte: Elaboração própria.

(1) O sinal da diferença média corresponde à subtração: média no $2^{\circ}$ momento - média no $1^{\circ}$ momento.

(2) Teste post-hoc de Bonferroni.

Foram analisados dados referentes apenas aos participantes do GI, estratificando-se esta análise conforme o tipo de participação na intervenção (individual e em grupo). Observou-se que, para aqueles que participaram da intervenção de forma individual, as médias para a pontuação de conhecimento sobre o diabetes foram diferentes entre o $1^{\mathrm{o}}$ e o $2^{\mathrm{o}}$ momento. Os resultados ainda mostram que as pontuações médias de autocuidado e ajustamento psicológico foram diferentes entre os tipos de participação na intervenção, no $2^{\text {o }}$ momento de observação (vide legenda da Tabela 4).

Tabela 4 - Síntese das comparações de pares entre momentos de observação e tipo de intervenção (covariável) junto a homens com Diabetes Mellitus tipo II para conhecimento, autocuidado, ajustamento psicológico e autoeficácia. Maringá, Paraná, Brasil - 2018. (N=30)

\begin{tabular}{|c|c|c|c|c|}
\hline \multirow{2}{*}{$\begin{array}{l}\text { Tipo de } \\
\text { Intervenção }\end{array}$} & \multicolumn{2}{|c|}{ Momento de observação } & \multirow{2}{*}{$\begin{array}{c}\text { Diferença média } \\
\text { (1) }\end{array}$} & \multirow{2}{*}{ p-valor (5) } \\
\hline & $1^{\circ}$ momento & $2^{\circ}$ momento & & \\
\hline \multicolumn{5}{|l|}{ Conhecimento } \\
\hline Individual & $9,09(2)$ & $10,68(3)$ & 1,59 & 0,005 \\
\hline Grupo & $9,18(2)$ & $10,28(3)$ & 1,10 & 0,109 \\
\hline \multicolumn{5}{|l|}{ Autocuidado } \\
\hline Individual & $3,75(2)$ & $3,58(4)$ & $-0,16$ & 0,575 \\
\hline Grupo & $3,69(2)$ & $4,28(4)$ & 0,59 & 0,099 \\
\hline \multicolumn{5}{|c|}{ Ajustamento psicológico } \\
\hline Individual & $48,31(2)$ & $45,81(4)$ & $-2,50$ & 0,450 \\
\hline Grupo & $51,14(2)$ & $51,92(4)$ & 0,78 & 0,789 \\
\hline
\end{tabular}


Tabela 4 - Síntese das comparações de pares entre momentos de observação e tipo de intervenção (covariável) junto a homens com Diabetes Mellitus tipo II para conhecimento, autocuidado, ajustamento psicológico e autoeficácia. Maringá, Paraná, Brasil - 2018. (N=30)

(conclusão)

\begin{tabular}{l|cc|c|c}
\hline \multirow{2}{*}{$\begin{array}{l}\text { Tipo de } \\
\text { Intervenção }\end{array}$} & \multicolumn{2}{|c|}{ Momento de observação } & Diferença média & \multirow{2}{*}{ p-valor(5) } \\
\cline { 2 - 5 } & $\mathbf{1}^{\mathbf{o}}$ momento & $\mathbf{2}^{\mathbf{o}}$ momento & $(1)$ & \\
\hline Autoeficácia & $3,78(2)$ & $3,78(3)$ & 0,00 & 0,993 \\
Individual & $3,80(2)$ & $3,81(3)$ & 0,01 & 0,954 \\
Grupo &
\end{tabular}

Fonte: Elaboração própria.

(1) O sinal da diferença média corresponde à subtração: média no $2^{\circ}$ momento - média no $1^{\circ}$ momento.

(2) Médias iguais dos Tipos de Intervenção no $1^{\circ}$ momento ( $\left.p>0,05\right)$.

(3) Médias iguais dos Tipos de Intervenção no $2^{\circ}$ momento ( $\left.p>0,05\right)$.

(4) Médias diferentes dos Tipos de Intervenção no $2^{\circ}$ momento $(\mathrm{p}<0,05)$.

(5) Teste post-hoc de Bonferroni.

Comparou-se ainda os resultados da participação individual e em grupo na intervenção com os resultados do GC e identificou-se que apenas no $2^{\circ}$ momento de observação ocorreram diferenças. Tanto os resultados da participação individual quanto do grupo foram diferentes do controle em relação ao conhecimento.

\section{Discussão}

A interação entre os grupos e os dois momentos distintos de observação apontou uma mudança significante no escore médio de conhecimento sobre o diabetes para os homens do GI, sinalizando o incremento nos níveis de conhecimento dos homens que participaram da intervenção de AA. Constatou-se ainda que a descontinuidade da intervenção podia implicar na estagnação ou diminuição dos níveis de conhecimento, o que ocorreu no GC.

Tal resultado corrobora o fato de que as intervenções provocaram melhora do conhecimento, o que se mostrou essencial, tendo em vista a cronicidade da condição e a necessidade de desenvolver habilidades. Estudo realizado com 52 pessoas de ambos os sexos, com idade média de 63,8 anos, mostrou aumento dos níveis satisfatórios de conhecimento de 51,9\% para 65,4\% após a intervenção conduzida por endocrinologista ${ }^{(16)}$, incremento menor, portanto, do que o observado na intervenção conduzida por enfermeiro no presente estudo.

Intervenção realizada durante seis meses, constituída por três consultas de enfermagem e contatos telefônicos mensais, também utilizou o AA. As 134 pessoas com DM participantes apresentaram melhora significativa sobre o escore médio do conhecimento sobre a doença, que passou de 6,0 para $9,0^{(3)}$.

Intervenções realizadas junto aos homens podem surtir bons resultados, como mostra estudo com homens trabalhadores metalúrgicos, com idade entre 18 e 70 anos, realizado no local de trabalho por três meses, em que foi observado acréscimo de 1,4 na escala de conhecimentos sobre doenças cardiovasculares ${ }^{(17)}$. O aumento foi um pouco maior do que o observado no presente estudo (1,1 ponto). Acredita-se que, no presente estudo, o aumento não foi maior porque os participantes já apresentavam, no momento inicial, um conhecimento considerado satisfatório.

Importa reforçar que o conhecimento por si só pode não ser suficiente para provocar mudanças efetivas no autocuidado. Isto aponta para a necessidade de intervenções que considerem as especificidades de gênero em suas abordagens. Sobre o autocuidado, o escore médio inicial dos homens em estudo já se apresentava maior do que o observado em outros ensaios clínicos ${ }^{(2-3)}$.

Intervenções realizadas durante 6 ou 12 meses, apresentaram, respectivamente, incrementos da ordem de $0,7^{(3)}$ a $0,9 \mathrm{dia}^{(2)}$ no número médio de dias de prática de autocuidado, o que leva à inferência de que, de forma geral, os homens participantes do presente estudo não foram beneficiados significativamente neste quesito. 
Isto, possivelmente, se deve ao pouco tempo de contato com o profissional enfermeiro, pois alguns usuários ainda hesitaram em reconhecer a sua responsabilidade acerca do autocuidado, de modo que ter alguém que pudesse apoiá-los seria importante ${ }^{(3)}$.

Contudo, no que tange aos homens, os enfermeiros enfrentam diversas dificuldades para implementar estratégias que convirjam para o atendimento das necessidades masculinas em saúde, sobretudo para sensibilizá-los quanto à importância de seu papel na gestão do autocuidado. Tais dificuldades perpassam, em boa parte, pelos desafios impostos por concepções de masculinidade que modulam o comportamento desse grupo social $^{(18)}$. No entanto, é necessário que os enfermeiros persistam na realização de intervenções de apoio ao autocuidado de homens com diabetes, especialmente em perspectiva interprofissional e colaborativa, pois, quando participam de intervenções dessa natureza, são identificados benefícios, como redução de fatores de risco cardiometabólicos ${ }^{(19)}$.

Sobre o ajustamento psicológico, não foram observados benefícios após a intervenção. Contudo, estudo realizado na Nigéria mostrou que o bom conhecimento sobre a doença e o tratamento, sobretudo acerca da importância da atividade física, podia favorecer o controle glicêmico, mesmo no caso de pessoas com uma atitude negativa diante da prática do exercício ${ }^{(20)}$.

Bons resultados sobre as atitudes relacionadas à doença foram apontados em estudo realizado junto a 238 indivíduos de ambos os sexos, sendo observado aumento significativo dos escores gerais relacionados às atitudes no grupo experimental $^{(2)}$. A baixa frequência de contato do pesquisador com os homens participantes do presente estudo pode ter limitado os avanços no tocante ao ajustamento psicológico, a ponto de interferir nas atitudes dos participantes diante da doença. Isto é, apesar das melhorias observadas em relação ao conhecimento, para ocorrer mudanças consolidadas de atitudes, seria fundamental a ocorrência de mais encontros entre esses e o enfermeiro.

No que concerne à autoeficácia, destaca-se ensaio clínico randomizado realizado no Sudeste brasileiro, com 183 pessoas com DM2 (GI=72), em que foi observada melhora nos escores de empoderamento ${ }^{(21)}$. É importante considerar a percepção da pessoa sobre sua autoeficácia, pois ela reflete suas crenças acerca das próprias capacidades e potencialidades para a produção do seu desempenho. As pessoas com percepção elevada de autoeficácia consideram tarefas que demandam muito esforço não como ameaças ou algo a ser evitado, mas sim como desafios que as motivam.

Contudo, como os homens participantes não desenvolveram, com a intervenção, atitudes significativamente positivas frente à doença, a percepção final que eles tiveram de suas capacidades para agir diante da alteração no seu estado de saúde ficou envolvida por um viés negativo. Assim, o atuar/agir desses homens ficou limitado, embora eles já tivessem um bom nível de conhecimento ou expressassem aquisição de novos conhecimentos ao longo do estudo. Isto, por sua vez, refletiu nos poucos avanços observados em relação às atividades/ações de autocuidado.

Ressalta-se, no caso desta pesquisa, que ligeiras diferenças em variáveis socioeconômicas, comportamentais e clínicas, embora não significantes, podiam representar relevância clínica. Ademais, apontaram para uma situação mais desfavorável dos homens que participaram do GI, o que podia determinar dificuldades para a obtenção de conhecimento, juízo crítico e tomada de decisão para o autocuidado.

Os homens, por vezes, apresentam dificuldades emocionais (medo, inquietação, raiva e tristeza) que refletem uma situação de afronta a uma masculinidade que os distancia do autocuidado em diabetes. Socialmente, o cuidado é vinculado ao universo feminino e, com isso, os homens recorrem tardiamente à mudança de comportamento ou à busca pelo cuidado profissional nos serviços de APS $^{(9)}$. Como parte desse cenário, eles podem deparar-se com episódios de agudização de sua condição, o que os leva a buscar serviços de pronto atendimento ${ }^{(22)}$. Há que se destacar a importância de os enfermeiros compreenderem os aspectos que estão relacionados à construção sociocultural dos homens com diabetes e que impactam sobre o cuidado em saúde ${ }^{(23)}$. 
Frente aos resultados observados, tanto na participação individual quanto em grupo, reforça-se que a intervenção provocou efeitos sobre o conhecimento, quando comparados os homens participantes do GC, independentemente do tipo de participação. Entretanto, no $2^{\circ}$ momento de observação, identificou-se diferença significante com escores maiores no autocuidado e ajustamento psicológico para os homens com participação em grupo.

Neste sentido, observa-se que a intervenção em grupo pode potencializar mudanças de hábito em homens, principalmente porque a relação de apoio estabelecida no grupo atua como elemento motivador para a implementação do autocuidado em condições crônicas, conforme estudo de intervenção educativa com metalúrgicos sobre fatores de risco para doenças cardiovasculares $^{(10)}$. Portanto, é importante que outros estudos com tamanhos amostrais maiores e com maior tempo e frequência de seguimento sejam desenvolvidos, visto que intervenções com programação específica de gênero podem resultar em achados contributivos para a prática clínica ${ }^{(24)}$.

Como limitações do estudo destacam-se as características de adesão da população-alvo, caracterizada por elevado índice de não comparecimento e de recusas em participar; considerável índice de perdas ao longo do seguimento, decorrente de desinteresse, e as falhas no protocolo de intervenção, pois era previsto um número maior de encontros e um contínuo acompanhamento telefônico. Estas limitações também podem guardar relação com o comportamento dos homens em relação à sua saúde, de se distanciar de intervenções profissionais, o que reforça a importância de se considerar a variável gênero no planejamento do cuidado de enfermagem voltado ao autogerenciamento em diabetes ${ }^{(25)}$.

Cabe destacar que o tipo de análise de dados empregada e o cuidado em se analisar possíveis diferenças nos dados quanto ao tipo de participação na intervenção configuraram-se em medidas oportunas para o controle de vieses relativos à seleção e às perdas no seguimento, produzindo resultados que permitiram identificar efeitos que, de fato, podem ser atribuídos ao AA.

O presente estudo contribui para a área da Enfermagem, pois apresenta resultados da avaliação de uma intervenção de AA dirigida exclusivamente a homens com DM2, implementada por um enfermeiro e sob condições de randomização e controle esperadas para o ensaio clínico. Evidencia, ao mesmo tempo, o potencial do AA e o desafio de se implementar intervenções junto à população masculina, pelo fato de existirem modos distintos de enfrentamento, influenciados por aspectos de gênero. Reforça a necessidade de estudos futuros minimizarem possíveis dificuldades no processo de intervenção, que expressem resultados adicionais e agreguem ao presente estudo na fundamentação de ações públicas oportunas para a saúde do homem em condição crônica.

\section{Conclusão}

A intervenção pautada no AA, conduzida por enfermeiro, produziu efeitos positivos e significantes sobre o nível de conhecimento de homens acerca do diabetes, especialmente na participação individual.

Sobre o tipo de participação, os homens da intervenção em grupo apresentaram melhor ajustamento psicológico e autocuidado do que aqueles da intervenção individual. Vale, portanto, destacar que resultados distintos podem ser encontrados, conforme a modalidade de participação no AA. Ao final do seguimento, verificou-se que o ajustamento psicológico do GC diminuiu significativamente, sugerindo que a ausência de intervenção pode ser negativa para a atitude dos homens frente ao diabetes. $\mathrm{Na}$ comparação entre os momentos inicial e final não foram observados efeitos significantes para os escores médios de autocuidado, ajustamento psicológico e autoeficácia.

\section{Colaborações:}

1 - concepção, projeto, análise e interpretação dos dados: Guilherme Oliveira de Arruda, 
Sonia Silva Marcon e Maria do Carmo Fernandez Lourenço Haddad;

2 - redação do artigo e revisão crítica relevante do conteúdo intelectual: Guilherme Oliveira de Arruda, Sonia Silva Marcon, Hellen Emília Peruzzo Aveiro, Maria do Carmo Fernandez Lourenço Haddad, Luciana Puchalski Kalinke, Gleiciane da Silva Fonseca e Afonso de Arruda Martinhago;

3 - aprovação final da versão a ser publicada: Guilherme Oliveira de Arruda, Sonia Silva Marcon, Hellen Emília Peruzzo Aveiro, Maria do Carmo Fernandez Lourenço Haddad, Luciana Puchalski Kalinke, Gleiciane da Silva Fonseca e Afonso de Arruda Martinhago.

\section{Referências}

1. International Diabetes Federation. Diabetes Atlas [Internet]. Brussels; 2017 [cited 2020 Aug 20]. Available from: https://www.idf.org/e-library/ epidemiology-research/diabetes-atlas/13-diabetesatlas-seventh-edition.html

2. Cortez DN, Macedo MML, Souza DAS, Santos JC, Afonso GS, Reis IA, et al. Evaluating the effectiveness of an empowerment program for self-care in type 2 diabetes: a cluster randomized trial. BMC Public Health. 2017;17(1):41. DOI: $10.1186 / \mathrm{s} 12889-016-3937-5$

3. Teston EF, Peternella FMN, Sale CA, Haddad MCL, Cubas MR, Marcon SS. Effect of the consultation of nursing on knowledge, quality of life, attitude towards disease and self-care among persons with diabetes. Rev Min Enferm. 2018;22:e-1106. DOI: $10.5935 / 1415-2762.20180034$

4. Funnell MM, Tang TS, Anderson RM. From DSME to DSMS: Developing EmpowermentBased Diabetes Self-Management Support. Diabetes Spect. 2007;20(4):221-6. DOI: 10.2337/ diaspect.20.4.221

5. Menezes MM, Lopes CT, Nogueira LS. Impact of educational interventions in reducing diabetic complications: a systematic review. Rev Bras Enferm. 2016;69(4):773-84. DOI: 10.1590/ 0034-7167.2016690422i

6. Arruda GO, Marcon SS. Health risk behaviors of men from the southern Brazil. Texto Contexto Enferm. 2018;27(2):e2640014. DOI: 10.1590/0104-070720180002640014
7. Rossaneis MA, Haddad MCFL, Mathias TAF, Marcon SS. Diferenças entre mulheres e homens diabéticos no autocuidado com os pés e estilo de vida. Rev Latino-Am Enfermagem. 2016;24:e2761. DOI: $10.1590 / 1518-8345.1203 .2761$

8. Hornung-Praehauser V, Ploessnig M, ZauchnerStudnicka S, Kautzky-Willer A, Leutner M. Better insight into gender-specific diabetes selfmanagement for more effective diabetes services. Eur J Public Health. 2016;26(suppl_1):278-9. DOI: $10.1093 /$ eurpub/ckw174.121

9. Sousa AR, Queiroz AM, Florencio RMS, Portela PP, Fernandes JD, Pereira A. Homens nos serviços de atenção básica à saúde: repercussões da construção social das masculinidades. Rev baiana enferm. 2016;30(3):1-10. DOI: 10.18471/ rbe.v30i3.16054

10. Marcon SS, Ganassin GS, Arruda GO, Teston EF, Barreto MS, Marquete $\mathrm{VF}$, et al. Intervenção educativa no local de trabalho: promoção da saúde e prevenção cardiovascular com homens metalúrgicos. Rev baiana enferm. 2021;35:e38619. DOI: https://doi.org/10.18471/rbe.v35.38619

11. Rosu MB, Oliffe JL, Kelly MT. Nurse Practitioners and Men's Primary Health Care. Am J Mens Health. 2017;11(5):1501-11. DOI: $10.1177 / 1557988315617721$

12. Hurt TR, Francis SL, Seawell AH, Krisco MP, Flynn MH, O'Connor MC, et al. Revising Diabetes Programming for Black Men and Their Families. Glob Qual Nurs Res. 2020;7:1-17. DOI: $10.1177 / 2333393620960183$

13. Arruda GO, Marcon SS, Peruzzo HE, Ruiz AG, Back IR, Nass EM, et al. Intervenção educativa em homens com diabetes mellitus: efeitos sobre comportamentos e perfil antropométrico. Acta Paul Enferm. 2020;33:eAPE20190128. DOI: 10.37689/acta-ape/2020AO0128

14. Curcio R, Lima MHM, Alexandre NMC. Instrumentos relacionados ao diabetes mellitus adaptados e validados para a cultura brasileira. Rev Eletr Enf. 2011;13(2):331-7. DOI: 10.5216/ree. v13i2.9476

15. Chaves FF, Reis IA, Pagano AS, Torres HC. Translation, cross-cultural adaptation and validation of the Diabetes Empowerment ScaleShort Form. Rev Saúde Pública. 2017;51(0):1-9. DOI: $10.1590 /$ s1518-8787.2017051006336

16. Eik Filho W, Bonjorno LP, Franco AJM, Santos MLA, Souza EM, Marcon SS. Evaluation, 
intervention, and follow-up of patients with diabetes in a primary health care setting in Brazil: the importance of a specialized mobile consultancy. Diabetol Metab Syndr. 2016;8:56. DOI: 10.1186/ s13098-016-0173-1

17. Ganassin GS, Silva EM, Pimenta AM, Marcon SS. Efficacy of an educative intervention on knowledge related to cardiovascular diseases among men. Acta Paul Enferm. 2016;29(1):38-46. DOI: $10.1590 / 1982-0194201600006$

18. Oliveira JA, Araújo IFM, Silva GTR, Sousa AR, Pereira A. Strategies and competences of nurses in men's health care: an integrative review. Rev Bras Enferm. 2020;73(Suppl 6):e20190546. DOI: http://dx.doi.org/10.1590/0034-7167-2019-0546

19. Lee JK, McCutcheon LRM, Fazel MT, Cooley JH, Slack MK. Assessment of Interprofessional Collaborative Practices and Outcomes in Adults with Diabetes and Hypertension in Primary Care: A Systematic Review and Meta-analysis. JAMA Netw Open. 2021;4(2):e2036725. DOI: 10.1001/ jamanetworkopen.2020.36725

20. Awotidebe TO, Adedoyin RA, Afolabi MA, Opiyo R. Knowledge, attitude and practice of exercise for plasma blood glucose control among patients with type-2 diabetes. Diabetes Metab Syndr. 2016;10(Suppl 1):S1-6. DOI: 10.1016/j. dsx.2016.01.006
21. Macedo MML, Cortez DN, Santos JC, Reis IA, Torres HC. Adherence to self-care practices and empowerment of people with diabetes mellitus: a randomized clinical trial. Rev Esc Enferm USP. 2017;51:e03278. DOI: 10.1590/ S1980-220X2016050303278

22. Silva JC, Nasif SS, Sousa AR, Santos SD, Mota TN, Pereira A. Características clínicas de homens atendidos em unidade de pronto atendimento. Rev baiana enferm. 2020;34:e34702. DOI: $10.18471 /$ rbe.v34.34702

23. Quaglia V. Men, masculinities and diabetes: 'doing gender' in Italian men's narratives of chronic illness. Sociol Health Illn. 2020;42(8):1902-17. DOI: $10.1111 / 1467-9566.13176$

24. Hawkins JM. Type 2 Diabetes Self-Management in Non-Hispanic Black Men: a Current State of the Literature. Curr Diab Rep. 2019 Feb 11;19(3):10. DOI: $10.1007 /$ s11892-019-1131-8

25. Alexandre K, Campbell J, Bugnon M, Henry C, Schaub C, Serex M, et al. Factors influencing diabetes self-management in adults: an umbrella review of systematic reviews. JBI Evid Synth. 2021;19(5):1003-18. DOI: 10.11124/JBIES-20-00020 Recebido: 8 de fevereiro de 2021 Aprovado: 22 de outubro de 2021 Publicado: 7 de dezembro de 2021

A Revista Baiana de Enfermagem utiliza a Licença Creative Commons - Atribuição-NãoComercial 4.0 Internacional. https://creativecommons.org/licenses/by-nc/4.0/ Este artigo é de acesso aberto distribuído sob os termos da Licença Creative Commons (CC BY-NC). Esta licença permite que outros remixem, adaptem e criem a partir do seu trabalho para fins não comerciais. Embora os novos trabalhos tenham de lhe atribuir o devido crédito e não possam ser usados para fins comerciais, os usuários não têm de licenciar esses trabalhos derivados sob os mesmos termos. 